\title{
Seasonal temperature variability and emergency hospital admissions for respiratory diseases: a population-based cohort study
}

\author{
Shengzhi Sun, ${ }^{1,2}$ Francine Laden, ${ }^{2,3}$ Jaime E Hart, ${ }^{2,3}$ Hong Qiu, ${ }^{1}$ Yan Wang, ${ }^{2}$ \\ Chit Ming Wong, ${ }^{1}$ Ruby Siu-yin Lee, ${ }^{4}$ Linwei Tian'
}

\begin{abstract}
- Additional material is published online only. To view please visit the journal online (http://dx.doi.org/10.1136/ thoraxjnl-2017-211333).

${ }^{1}$ School of Public Health, Li Ka Shing Faculty of Medicine, The University of Hong Kong, Hong Kong Special Administrative Region, Hong Kong

${ }^{2}$ Department of Environmental Health, Harvard T.H. Chan School of Public Health, Boston, Massachusetts, USA ${ }^{3}$ Channing Division of Network Medicine, Department of Medicine, Brigham and Women's Hospital and Harvard Medical School, Boston,

Massachusetts, USA

${ }^{4}$ Elderly Health Service, Department of Health, Hong Kong Special Administrative Region, Hong Kong
\end{abstract}

Correspondence to Dr Linwei Tian, School of Public Heath, Li Ka Shing Faculty of Medicine, The University of Hong Kong, Pokfulam, Hong

Received 28 November 2017 Revised 5 March 2018 Accepted 26 March 2018 Published Online First 5 April 2018 Kong; linweit@hku.hk

\begin{abstract}
Background Climate change increases global mean temperature and changes short-term (eg, diurnal) and long-term (eg, intraseasonal) temperature variability. Numerous studies have shown that mean temperature and short-term temperature variability are both associated with increased respiratory morbidity or mortality. However, data on the impact of long-term temperature variability are sparse.

Objective We aimed to assess the association of intraseasonal temperature variability with respiratory disease hospitalisations among elders.

Methods We ascertained the first occurrence of emergency hospital admissions for respiratory diseases in a prospective Chinese elderly cohort of 66820 older people ( $\geq 65$ years) with 10-13 years of follow-up. We used an ordinary kriging method based on 22 weather monitoring stations in Hong Kong to spatially interpolate daily ambient temperature for each participant's residential address. Seasonal temperature variability was defined as the SD of daily mean summer (June-August) or winter (December-February) temperatures. We applied Cox proportional hazards regression with timevarying exposure of seasonal temperature variability to respiratory admissions.
\end{abstract}

Results During the follow-up time, we ascertained 12 689 cases of incident respiratory diseases, of which 6672 were pneumonia and 3075 were COPD. The HRs per $1{ }^{\circ} \mathrm{C}$ increase in wintertime temperature variability were 1.20 (95\% Cl 1.08 to 1.32$), 1.15$ (1.01 to 1.31 ) and 1.41 (1.15 to 1.71) for total respiratory diseases, pneumonia and $C O P D$, respectively. The associations were not statistically significant for summertime temperature variability.

Conclusion Wintertime temperature variability was associated with higher risk of incident respiratory diseases.

\section{INTRODUCTION}

SLinked

- http://dx.doi.org/10.1136/ thoraxjnl-2018-211898

Check for updates

To cite: Sun S, Laden F, Hart JE, et al. Thorax 2018:73:951-958.
Respiratory diseases, including pneumonia and COPD, contribute to substantial health burden worldwide. Pneumonia affects approximately 450 million people a year and is a leading cause of hospitalisation and death worldwide. ${ }^{1} \mathrm{~A}$ total of 3.2 million people died of COPD in 2015, which is the fourth leading cause of death in the world. ${ }^{2}$

It is now well recognised that climate change has increased global temperature over the past few decades, primarily due to the increased emissions of

\section{Key messages}

What is the key question?

- Is seasonal temperature variability associated with increased risk of respiratory disease hospitalisations among elders?

What is the bottom line?

- Wintertime temperature variability was associated with higher risks of incidence of total respiratory diseases, pneumonia and COPD and such associations were stronger in females and participants in a lower socialeconomic position.

Why read on?

- This large prospective cohort study is the first to show the impact of seasonal temperature variability on respiratory diseases admissions, and it highlights the potential role of changing seasonal temperature variability introduced by climate change on respiratory system.

anthropogenic greenhouse gases. ${ }^{3}$ Climate change is also projected to cause changes in the frequency, severity and duration of extreme weather events, including changing temperature variability in shortterm (eg, diurnal temperature range) and long-term (eg, intraseasonal) ways. ${ }^{4-7}$ It is expected that climate change will affect respiratory diseases significantly through influencing viral activity and transmission (eg, respiratory syncytial virus), altering vectors and the host immune response and changing in allergen disposition. ${ }^{89}$

Mean and variability are two main characteristics of temperature. Numerous time-series and case-crossover studies have reported that shortterm exposure to both cold and hot temperatures were associated with increased risks of respiratory mortality ${ }^{10}$ and hospital admissions. ${ }^{11}$ Most of those studies focused on the adverse health effects of short-term mean temperature. A few studies also assessed the health effects of short-term temperature variability (eg, diurnal temperature range and temperature change between neighbouring days or diurnal temperature range). For example, Lim et $a l^{12}$ reported that diurnal temperature range was significantly associated with respiratory hospitalisations using four metropolitan areas in Korea with 
a total population of 18.3 million. To the best of knowledge, no study has been conducted to investigate the impacts of seasonal (long-term) temperature variability on incident respiratory disease hospital admissions.

The present study aimed to estimate the association between seasonal temperature variability, the SD of daily mean summer (June-August) or winter (December-February) temperatures and the incidence of respiratory disease hospital admissions in a prospective Chinese elderly cohort in Hong Kong. We also assessed whether the associations were modified by age, sex, marital and socioeconomic status and housing type to identify vulnerable subpopulations.

\section{METHODS}

\section{Study population}

The Chinese elderly cohort in Hong Kong is a prospective cohort, into which all residents of Hong Kong aged 65 years or older ( $\geq 65$ years) were eligible to enrol. From 1998 to 2001, 66 820 elders, about 9\% of older people in Hong Kong, enrolled in the 18 Elderly Health Centres of the Department of Health, one in each of the 18 districts in Hong Kong, and were followed up until December 2010. Each participant had physical examinations and face-to-face interviews by registered nurses or doctors using a standardised structured questionnaire during each year of follow-up. ${ }^{13}$ The collected information included demographic characteristics (eg, age and sex), socioeconomic status (eg, personal monthly expenditure), lifestyle (eg, smoking status and physical activity) and body mass index (BMI). Details of this cohort profile were described elsewhere. ${ }^{13}$

\section{Health outcomes}

We used a common unique identifier (the Hong Kong identity card number) to link the cohort with the Hospital Authority Corporate Data Warehouse, which covers all publicly funded hospitals that provide 24-hour accident and emergency services and covers $90 \%$ of hospital beds for Hong Kong residents. ${ }^{14}$ Hospital admissions were identified using primary discharge diagnoses for emergency hospital admissions for respiratory diseases (International Classification of Diseases, Ninth Revision: 460:519), pneumonia $(480: 486,487.0)$ and COPD (490:492, 494:496). Incident cases for respiratory diseases were ascertained as the first occurrence of emergency hospital admissions after enrolment.

\section{Ambient temperature}

We extracted daily mean ambient temperature data from the 22 weather monitoring stations in Hong Kong within a land area of $1104 \mathrm{~km}^{2}$ from 1998 to 2010 (figure 1). Among various algorithms (eg, kriging, inverse distance weighting and trend surface

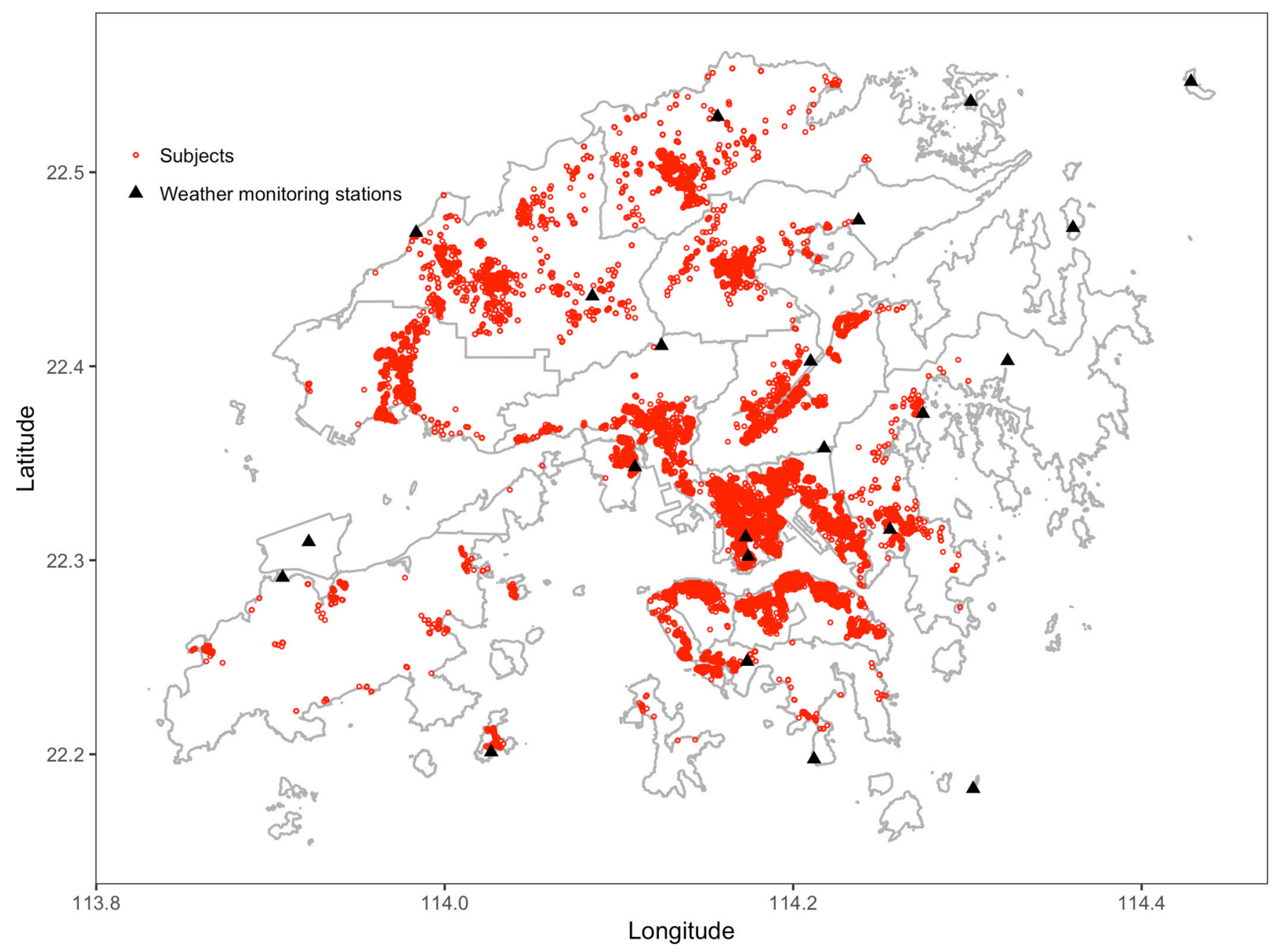

Figure 1 Locations of participants in the prospective Chinese elderly cohort $(n=61446)$ at baseline (1998-2001) and weather monitoring stations $(n=22)$ in Hong Kong. 
analysis) to spatial interpolate daily mean ambient temperature, the kriging method yields a more realistic spatial behaviour of the climatological variable of interest. ${ }^{15}{ }^{16}$ Ordinary kriging is the most commonly used kriging method, which estimates daily temperature at locations without monitors based on the weighted average of adjacent observed sites within a given area. Ordinary kriging has been described as the 'anchor algorithm of geostatistics' because of its remarkable robustness under a range of conditions. ${ }^{17}$ We used ordinary kriging to interpolate the daily ambient temperature based on the 22 weather monitoring stations for each participant according to his or her residential address. The performance of the ambient temperature prediction model was validated by leave-one-out cross-validation. The $\mathrm{R}^{2}$ of leave-one-out cross-validation was found to be very high $\left(\mathrm{R}^{2}=0.93\right)$. We then calculated the SD of daily mean summer (June-August) temperature (summertime temperature variability) and the SD of daily mean winter (December-February) temperature (wintertime temperature variability) for each participant's residential address. High or low temperature variability was dichotomously defined as higher or lower than the median of seasonal temperature variability.

\section{Fine particulate matter $\left(\mathrm{PM}_{2.5}\right)$ exposure}

We estimated $\mathrm{PM}_{2.5}$ exposure based on Surface Extinction Coefficients (SEC) from aerosol optical Depth (AOD) retrieved from remote sensing data of the two National Aeronautics and Space Administration Earth Observing System satellites. ${ }^{18}$ AOD data were originally retrieved at a $10 \times 10 \mathrm{~km}$ resolution and were refined into $1 \times 1 \mathrm{~km}$ resolution by modifying the Moderate Resolution Imaging Spectroradiometer algorithm. ${ }^{19}$ The relationship between SEC and $\mathrm{PM}_{25}$ for each year from 1998 to 2010 was calibrated using grid cells with both SEC and PM $_{25}$ measurements. This yearly calibration was then used to estimate $\mathrm{PM}_{2.5}$ at the residential location of each participant. The estimated $\mathrm{PM}_{2.5}$ concentrations have been used in earlier studies in this cohort to reveal the association of $\mathrm{PM}_{25}$ with mortality or hospital admissions. ${ }^{20-22}$

\section{Individual and ecological covariates}

We controlled for individual-level potential confounders, including age, sex, marital status, BMI, physical activity, housing type, education attainment, smoking status, medication taken and personal monthly expenditure. As tertiary planning units (TPUs) are the most commonly used units in the population census report in Hong Kong, we calculated the social deprivation index (SDI) to control for TPU-level social deprivation. Details for the calculation of SDI were described elsewhere. ${ }^{23} 24$ We also controlled for smoking rate ( $>15$ years of age) at the district level.

\section{Statistical analysis}

We used Cox proportional hazards models to estimate the association between seasonal temperature variability and incident respiratory diseases. Survival time was calculated from enrolment date to the first hospital admission to respiratory diseases or death or 31 December 2010 (censoring), whichever came first. In order to separate the independent effects of summertime or wintertime temperature variability, we included yearly mean temperature and summertime and wintertime temperature variability simultaneously in the model and treated them as time-varying exposures. To do this, we used the counting process approach to the Cox proportional hazards model. ${ }^{25} \mathrm{We}$ controlled for individual-, TPU- and district-level risk factors as mentioned above and included a linear term for year of follow-up to adjust for time trends. To allow for possible non-proportionality of hazard, age in years was treated as the stratification variable. We also examined age ( $\leq 70$ years and $>70$ years), sex (female and male), marital status (married and unmarried), education attainment (below primary, primary and secondary or above), personal monthly expenditure (low, medium and high) and housing type (public and aided, private and others) as the potential modifiers of the effects of seasonal temperature variability by including an interaction term between seasonal temperature variability and one effect modifier at a time in the model, and $\mathrm{p}$ value of the interaction term was used to indicate statistical significance.

We performed a number of sensitivity analyses to test the robustness of our results. First, we used inverse distance weighting to spatially interpolate daily temperature for each participant, and then refitted the Cox proportional hazards regression model. Second, to control for competing diseases, we excluded participants who died or who had incidence of respiratory diseases during the first year after enrolment or excluded those who self-reported COPD/asthma at the baseline. Third, considering $\mathrm{PM}_{2.5}$ concentration may vary from year to year, we controlled for time-varying $\mathrm{PM}_{2.5}$ in addition to all potential confounders mentioned above. We did not control for $\mathrm{PM}_{2.5}$ in the main analysis as the role of air pollution on the association between ambient temperature and morbidity or mortality is complex and has not be fully elucidated. ${ }^{26}$ Fourth, participants might move their homes during the 10-13 years of follow-up, so we excluded participants who changed their home addresses. Finally, we tested the assumption of linearity for seasonal temperature variability by using natural cubic spline functions with three $\mathrm{df}$.

The results were expressed as HR per $1^{\circ} \mathrm{C}$ increase in seasonal temperature variability. All analyses were conducted in $R$ statistical environment V.3.3.0, with packages 'geoR' to interpolate individual's daily ambient temperature using ordinary kriging methods and 'survival' for survival analysis to estimate the HR.

\section{RESULTS}

A total of 66820 older people were enrolled in the initial study cohort. After excluding participants without sufficient address information for geocoding or with missing covariates, a final sample of $61446(92.0 \%)$ was included in the final analyses. The spatial distribution of these 61446 older people is shown in figure 1.

Table 1 shows the baseline descriptive characteristics of the 61446 participants included in this study. The mean age at entry of this cohort was 72 years old and female participants accounted for 65.9\%. A total of 14168 (23.1\%) participants' BMI were less than 21.6, and 16100 (26.2\%) participants' BMI were greater than 26.3 . The majority of participants did physical activity seven days/week. About one-fifth (19.3\%) were former smokers, and $9.6 \%$ were current smokers. About half of the participants $(46.0 \%)$ had education attainment below primary. Nearly half of the participants (53.1\%) took regular medication. Participants who were exposed to a higher wintertime temperature variability were more likely to be older, female, smokers and have lower personal monthly expenditure but less likely to have a secondary or higher education and live in private house when compared with those exposed to a lower wintertime temperature variability (table 1). There were no apparent longterm trends for yearly mean and seasonal mean temperatures 
Table 1 Descriptive characteristics of the prospective Chinese elderly cohort at baseline (1998-2001) by seasonal temperature variability

\begin{tabular}{|c|c|c|c|c|c|}
\hline \multirow[b]{4}{*}{ Characteristic } & \multirow[b]{4}{*}{ Total } & \multirow{2}{*}{\multicolumn{2}{|c|}{$\begin{array}{l}\text { Summertime temperature } \\
\text { variability* } \\
\text { (Mean } \pm \text { SD: } 1.4 \pm 0.2 \text { ) }\end{array}$}} & \multirow{2}{*}{\multicolumn{2}{|c|}{$\begin{array}{l}\text { Wintertime temperature } \\
\text { variability } t \\
\text { (Mean } \pm \text { SD: } 3.2 \pm 0.5 \text { ) }\end{array}$}} \\
\hline & & & & & \\
\hline & & Low & High & Low & High \\
\hline & & $(1.2 \pm 0.1)$ & $(1.6 \pm 0.1)$ & $(3.0 \pm 0.2)$ & $(3.4 \pm 0.1)$ \\
\hline Participants, n & 61446 & 30723 & 30723 & 30722 & 30724 \\
\hline \multicolumn{6}{|l|}{ Individual-level covariates } \\
\hline \multicolumn{6}{|l|}{$\begin{array}{l}\text { Age at entry, year, } \\
\mathrm{n}(\%)\end{array}$} \\
\hline$\leq 70$ & $29038(47.3)$ & $14450(47.0)$ & $14588(47.5)$ & $14848(48.3)$ & $14190(46.2)$ \\
\hline$>70$ & $32408(52.7)$ & $16273(53.0)$ & $16135(52.5)$ & $15874(51.7)$ & $16534(53.8)$ \\
\hline \multicolumn{6}{|l|}{ Sex, n (\%) } \\
\hline Male & $20933(34.1)$ & $10384(33.8)$ & $10549(34.3)$ & 10930 (35.6) & $10003(32.6)$ \\
\hline Female & $40513(65.9)$ & $20339(66.2)$ & $20174(65.7)$ & $19792(64.4)$ & $20721(67.4)$ \\
\hline \multicolumn{6}{|l|}{ BMI quartiles, n (\%) } \\
\hline 1 st $(<21.6)$ & $14168(23.1)$ & $7100(23.1)$ & $7068(23.0)$ & $7143(23.3)$ & 7025 (22.9) \\
\hline 2 nd- $3 r d(21.6-26.3)$ & $31178(50.7)$ & $15574(50.7)$ & $15604(50.8)$ & $15616(50.8)$ & $15562(50.7)$ \\
\hline 4th $(>26.3)$ & $16100(26.2)$ & $8049(26.2)$ & $8051(26.2)$ & $7963(25.9)$ & $8137(26.5)$ \\
\hline \multicolumn{6}{|l|}{ Smoking status, n (\%) } \\
\hline Never & $43668(71.1)$ & $21946(71.4)$ & $21722(70.7)$ & $22058(71.8)$ & $21610(70.3)$ \\
\hline Former & $11871(19.3)$ & $5830(19.0)$ & $6041(19.7)$ & 5791 (18.8) & $6080(19.8)$ \\
\hline Current & $5907(9.6)$ & $2947(9.6)$ & $2960(9.6)$ & $2873(9.4)$ & $3034(9.9)$ \\
\hline \multicolumn{6}{|l|}{$\begin{array}{l}\text { Exercise in days/week, } \\
n(\%)\end{array}$} \\
\hline Never (0) & $9406(15.3)$ & $4667(15.2)$ & 4739 (15.4) & $4784(15.6)$ & $4622(15.0)$ \\
\hline Medium (1-6) & 7788 (12.7) & $3996(13.0)$ & $3792(12.3)$ & $4172(13.6)$ & $3616(11.8)$ \\
\hline High (7) & $44252(72.0)$ & $22060(71.8)$ & $22192(72.2)$ & $21766(70.8)$ & $22486(73.2)$ \\
\hline \multicolumn{6}{|l|}{$\begin{array}{l}\text { Education attainment, } \\
\mathrm{n}(\%)\end{array}$} \\
\hline Below primary & $28241(46.0)$ & $14201(46.2)$ & $14040(45.7)$ & $13268(43.2)$ & $14973(48.7)$ \\
\hline Primary & 22656 (36.9) & $11434(37.2)$ & 11222 (36.5) & $11162(36.3)$ & 11494 (37.4) \\
\hline Secondary or above & $10549(17.2)$ & $5088(16.6)$ & $5461(17.8)$ & $6292(20.5)$ & $4257(13.9)$ \\
\hline \multicolumn{6}{|l|}{ Housing type, n (\%) } \\
\hline Private & $32458(52.8)$ & $16359(53.2)$ & $16099(52.4)$ & $18239(59.4)$ & $14219(46.3)$ \\
\hline Public and aided & $25096(40.8)$ & $12503(40.7)$ & $12593(41.0)$ & $10664(34.7)$ & $14432(47.0)$ \\
\hline Other & $3892(6.3)$ & $1861(6.1)$ & $2031(6.6)$ & $1819(5.9)$ & $2073(6.7)$ \\
\hline \multicolumn{6}{|l|}{$\begin{array}{l}\text { Expenses/month in US\$, } \\
\mathrm{n}(\%)\end{array}$} \\
\hline Low $(<128)$ & $10122(16.5)$ & $5021(16.3)$ & $5101(16.6)$ & $5169(16.8)$ & $4953(16.1)$ \\
\hline Medium (128-384) & 42151 (68.6) & $21092(68.7)$ & $21059(68.5)$ & $20576(67.0)$ & $21575(70.2)$ \\
\hline High ( $\geq 385)$ & $9173(14.9)$ & $4610(15.0)$ & $4563(14.9)$ & 4977 (16.2) & $4196(13.7)$ \\
\hline \multicolumn{6}{|l|}{ Medication taken, $\mathrm{n}(\%)$} \\
\hline Yes & $32627(53.1)$ & $16397(53.4)$ & $16230(52.8)$ & $16430(53.5)$ & $16197(52.7)$ \\
\hline No & $28819(46.9)$ & $14326(46.6)$ & $14493(47.2)$ & $14292(46.5)$ & $14527(47.3)$ \\
\hline \multicolumn{6}{|l|}{ TPU-level covariate } \\
\hline SDI $($ mean $\pm S D)$ & $13.8(2.0)$ & $13.9(2.1)$ & $13.8(2.0)$ & $14.0(2.3)$ & $13.7(1.8)$ \\
\hline \multicolumn{6}{|l|}{ District-level covariate } \\
\hline Smoking rate $($ mean $\pm S D)$ & $11.6(0.4)$ & $11.6(0.3)$ & $11.5(0.4)$ & $11.6(0.3)$ & $11.5(0.4)$ \\
\hline
\end{tabular}

${ }^{*}$ High and low summertime temperature variability was defined by the median $\left(1.4^{\circ} \mathrm{C}\right)$ of the $S D$ of daily mean summer temperature. tHigh and low wintertime temperature variability was defined by the median $\left(3.2^{\circ} \mathrm{C}\right)$ of the $S D$ of daily mean winter temperature. $\mathrm{BMI}$, body mass index; SDI, social deprivation index; TPUs, tertiary planning units. 

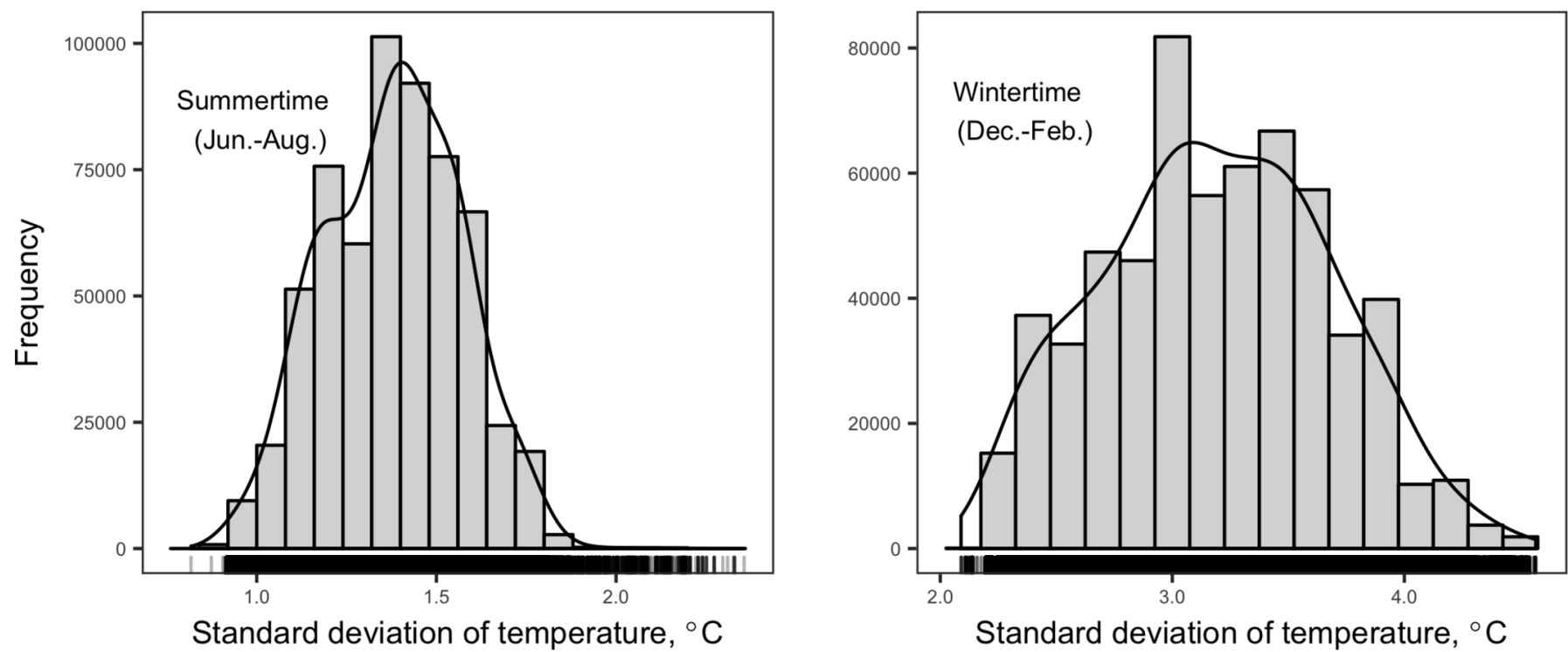

Figure 2 Distribution of the SD of daily mean summer and winter temperatures.

from 1998 to 2010 based on temperatures monitored by the 22 weather stations (online supplementary figure S1).

During the study period, summertime and wintertime temperature variability both approximated a normal distribution (figure 2) with mean SDs of $1.4^{\circ} \mathrm{C}$ and $3.2^{\circ} \mathrm{C}$ for summertime and wintertime, respectively. After 10-13 years of follow-up (from 1998 to 2010), there were 12689 emergency hospital admissions for respiratory diseases, among them pneumonia and COPD accounted for 52.6\% (6672) and $24.2 \%$ (3075), respectively (table 2).

\section{Seasonal temperature variability and incident respiratory diseases}

The associations of summertime and wintertime temperature variability with total incident respiratory diseases, pneumonia and COPD were presented in table 2 . In the basic models stratified by age in years and adjusted for sex and year of follow-up, summertime temperature variability was not associated with increased risks of total incident respiratory diseases, pneumonia or COPD. The associations between wintertime temperature variability and incident respiratory diseases were all statistically significant. The HRs were modestly attenuated for summertime temperature variability in the fully adjusted models with additional adjustment for yearly mean temperature, marital status, housing type, BMI, education attainment, personal monthly expenditure, physical activity, medication taken, smoking status, SDI and smoking rate at the district level. For example, the fully adjusted HR for total respiratory diseases was 1.02 (0.80 to 1.29) compared with 1.12 (0.91 to 1.39 ) in the basic model. Results for wintertime temperature variability were also modestly attenuated in the full models but remained statistically significant. The HR was 1.20 (1.08 to 1.32) for total incident respiratory diseases, 1.15 (1.01 to 1.31 ) for pneumonia and 1.41 (1.15 to 1.71) for COPD per $1^{\circ} \mathrm{C}$ change in wintertime temperature variability in the fully adjusted models.

In this Hong Kong elderly cohort, about $11.1 \%$ elders changed home addresses during the follow-up period. Excluding those participants from the analysis did not change the Cox regression results substantially (online supplementary table S1). Sensitivity analyses excluding participants according to other criteria or further controlling for time-varying $\mathrm{PM}_{2.5}$ exposure gave similar results (table 3). We also used inverse distance weighting to spatially interpolate daily temperature for each participant and refitted the Cox proportional hazards regression, and we found results were similar to those using the ordinary kriging method (online supplementary table S2). By comparing the linear and natural cubic spline models, we did not find any evidence of departure from linearity for the association between seasonal temperature variability and respiratory diseases hospitalisations.

\section{Effect modification for seasonal temperatures variability}

We examined associations of seasonal temperature variability with incident respiratory diseases in the fully adjusted models stratified by age ( $\leq 70$ years and $>70$ years), sex (female and male), marital status (married and unmarried), education attainment (below primary, primary and secondary or above), personal

Table $2 \mathrm{HR}$ and $95 \% \mathrm{Cl}$ per $1{ }^{\circ} \mathrm{C}$ increase of seasonal temperature variability on incident respiratory diseases in the prospective Chinese elderly cohort in Hong Kong

\begin{tabular}{|c|c|c|c|c|c|}
\hline \multirow[b]{2}{*}{ Incident diseases } & \multirow[b]{2}{*}{ Cases } & \multicolumn{2}{|c|}{ Summertime temperature variability } & \multicolumn{2}{|c|}{ Wintertime temperature variability } \\
\hline & & Basic model* & Full modelt & Basic model* & Full modelt \\
\hline Total respiratory diseases & 12689 & 1.12 (0.91 to 1.39 ) & 1.02 (0.80 to 1.29$)$ & 1.50 (1.39 to 1.62$)$ & 1.20 (1.08 to1.32) \\
\hline Pneumonia & 6672 & 1.06 (0.78 to 1.43$)$ & 1.04 (0.75 to 1.44$)$ & $1.31(1.18$ to 1.46$)$ & $1.15(1.01$ to 1.31$)$ \\
\hline COPD & 3075 & 1.14 (0.75 to 1.74$)$ & 1.05 (0.65 to 1.69$)$ & 1.96 (1.67 to 2.30$)$ & 1.41 (1.15 to 1.71$)$ \\
\hline
\end{tabular}

*Stratified by age in years, adjusting for sex and year of follow-up and summertime temperature variability and wintertime temperature variability were mutually adjusted.

†Additionally adjusted for yearly mean temperature, marital status, housing type, BMI, education attainment, personal monthly expenditure, physical activity, medication taken, smoking status, social deprivation index and smoking rate at the district level.

BMI, body mass index. 
Table $3 \mathrm{HR}$ and $95 \% \mathrm{Cl}$ per $1{ }^{\circ} \mathrm{C}$ increase of seasonal temperature variability on incident respiratory diseases in the sensitivity analyses

\begin{tabular}{|c|c|c|c|c|c|}
\hline \multirow[b]{2}{*}{ Incident diseases } & \multirow[b]{2}{*}{ Cases } & \multicolumn{2}{|c|}{ Summertime temperature variability } & \multicolumn{2}{|c|}{ Wintertime temperature variability } \\
\hline & & Basic model* & Full modelt & Basic model* & Full modelt \\
\hline \multicolumn{6}{|c|}{ Excluding those with incident respiratory diseases or died in the first year } \\
\hline Total respiratory diseases & 12110 & 1.11 (0.89 to 1.38$)$ & 1.01 (0.79 to 1.30$)$ & $1.47(1.36$ to 1.60$)$ & $1.18(1.07$ to 1.31$)$ \\
\hline Pneumonia & 6591 & 1.07 (0.79 to 1.45$)$ & 1.06 (0.76 to 1.48$)$ & 1.31 (1.18 to 1.46$)$ & $1.16(1.01$ to 1.32$)$ \\
\hline COPD & 2877 & 1.14 (0.73 to 1.76$)$ & 1.05 (0.64 to 1.71$)$ & 1.92 (1.63 to 2.26$)$ & $1.36(1.11$ to 1.66$)$ \\
\hline \multicolumn{6}{|c|}{ Excluding those with self-reported COPD/asthma at baseline } \\
\hline Total respiratory diseases & 10794 & $1.02(0.81$ to 1.29$)$ & 0.96 (0.74 to 1.25$)$ & 1.44 (1.32 to 1.57$)$ & $1.17(1.05$ to 1.30$)$ \\
\hline Pneumonia & 5890 & 1.10 (0.80 to 1.52$)$ & 1.12 (0.79 to 1.59$)$ & 1.28 (1.14 to 1.43$)$ & $1.13(0.98$ to 1.31$)$ \\
\hline COPD & 1896 & 1.07 (0.62 to 1.85$)$ & $1.03(0.56$ to 1.90$)$ & 1.78 (1.45 to 2.17$)$ & $1.30(1.01$ to 1.67$)$ \\
\hline \multicolumn{6}{|c|}{ Further adjusted for time-varying $\mathrm{PM}_{2.5}$} \\
\hline Total respiratory diseases & 12689 & 1.12 (0.91 to 1.39$)$ & 1.08 (0.84 to 1.40$)$ & 1.50 (1.39 to 1.62$)$ & 1.18 (1.06 to 1.31$)$ \\
\hline Pneumonia & 6672 & 1.06 (0.78 to 1.43$)$ & 1.07 (0.75 to 1.52$)$ & 1.31 (1.18 to 1.46$)$ & $1.17(1.02$ to 1.34$)$ \\
\hline COPD & 3075 & 1.14 (0.75 to 1.74$)$ & 1.15 (0.69 to 1.92$)$ & 1.96 (1.67 to 2.30$)$ & $1.41(1.15$ to 1.74$)$ \\
\hline
\end{tabular}

*Stratified by age in years, adjusting for sex and year of follow-up and summertime temperature variability and wintertime temperature variability were mutually adjusted. tAdditionally adjusted for yearly mean temperature, marital status, housing type, BMI, education attainment, personal monthly expenditure, physical activity, medication taken, smoking status, social deprivation index and smoking rate at the district level.

$\mathrm{BMI}$, body mass index; $\mathrm{PM}_{2.5^{\prime}}$ particulate matter with an aerodynamic diameter $\leq 2.5 \mu \mathrm{m}$.

monthly expenditure (low, medium and high) and housing type (public and aided, private and others). No significant difference was observed by stratified characteristics, except for sex and personal monthly expenditure for the wintertime temperature variability (table 4). Female and those with lower personal monthly expenditures had greater HRs when exposed to wintertime temperature variability. Female also exhibited increased risk of respiratory hospitalisations after exposure to summertime temperature variability (online supplementary table S3).

\section{DISCUSSION}

We found associations between wintertime temperature variability and total incident respiratory diseases, pneumonia

Table $4 \mathrm{HR}$ and $95 \% \mathrm{Cl}$ per $1^{\circ} \mathrm{C}$ increase in wintertime temperature variability stratified by population characteristics in the prospective Chinese elderly cohort in Hong Kong, 1998-2010

\begin{tabular}{|c|c|c|c|c|c|c|c|c|c|}
\hline \multirow{2}{*}{$\begin{array}{l}\text { Stratified } \\
\text { characteristics }\end{array}$} & \multicolumn{3}{|c|}{ Total respiratory $(n=12689)$} & \multicolumn{3}{|c|}{ Pneumonia $(n=6672)$} & \multicolumn{3}{|c|}{ COPD $(n=3075)$} \\
\hline & Cases & HR $(95 \% \mathrm{Cl})$ & $\mathbf{P}_{\text {Interaction }}$ & Cases & HR $(95 \% \mathrm{Cl})$ & $\mathbf{P}_{\text {Interaction }}$ & Cases & $\mathrm{HR}(95 \% \mathrm{Cl})$ & $\mathbf{P}_{\text {Interaction }}$ \\
\hline \multicolumn{10}{|l|}{ Age at entry, year } \\
\hline$>70$ & 9093 & $1.17(1.05$ to 1.30$)$ & 0.28 & 5073 & 1.12 (0.97 to 1.28$)$ & 0.19 & 2220 & 1.38 (1.12 to 1.71$)$ & 0.69 \\
\hline \multicolumn{10}{|l|}{ Sex } \\
\hline \multicolumn{10}{|l|}{ Marital status } \\
\hline Unmarried & 6802 & $1.20(1.09$ to 1.34$)$ & & 3607 & $1.17(1.02$ to 1.35$)$ & & 1536 & 1.46 (1.18 to 1.80$)$ & \\
\hline Married & 5887 & 1.19 (1.07 to 1.32$)$ & 0.68 & 3065 & $1.13(0.98$ to 1.30$)$ & 0.40 & 1539 & 1.35 (1.09 to 1.68$)$ & 0.34 \\
\hline \multicolumn{10}{|l|}{ Education attainment } \\
\hline \multicolumn{10}{|l|}{ Housing type } \\
\hline Public and aided & 5544 & 1.19 (1.07 to 1.33$)$ & & 2862 & 1.22 (1.05 to 1.41$)$ & & 1403 & 1.43 (1.14 to 1.79$)$ & \\
\hline Private & 5714 & $1.22(1.10$ to 1.35$)$ & 0.53 & 3002 & 1.11 (0.97 to 1.28$)$ & 0.06 & 1340 & 1.43 (1.16 to 1.77$)$ & 0.98 \\
\hline Other & 1431 & 1.15 (1.00 to 1.32$)$ & 0.56 & 808 & 1.15 (0.96 to 1.37$)$ & 0.44 & 332 & 1.27 (0.96 to 1.69$)$ & 0.37 \\
\hline \multicolumn{10}{|l|}{ Expenses/money in US\$ } \\
\hline Low $(<128)$ & 1857 & $1.32(1.16$ to 1.50$)$ & & 1001 & 1.25 (1.05 to 1.48$)$ & & 427 & 1.34 (1.02 to 1.75$)$ & \\
\hline Medium (128-384) & 8700 & $1.18(1.07$ to 1.31$)$ & 0.03 & 4508 & $1.14(1.00$ to 1.31$)$ & 0.17 & 2181 & 1.45 (1.19 to 1.78$)$ & 0.43 \\
\hline High ( $\geq 385)$ & 2132 & 1.17 (1.03 to 1.33$)$ & 0.06 & 1163 & 1.12 (0.95 to 1.32$)$ & 0.18 & 467 & 1.22 (0.93 to 1.59$)$ & 0.51 \\
\hline
\end{tabular}


and COPD among a prospective Chinese elderly cohort that accounted for about $9 \%$ of all elders in Hong Kong. Such associations were stronger in females and participants in a lower socialeconomic position. We did not find any association between summertime temperature variability and incident respiratory diseases.

In the literature, temperature variability within a day or a few neighbouring days has been consistently associated with respiratory mortality or hospitalisations. ${ }^{127-30}$ For example, diurnal temperature change was generally associated with emergency hospital admissions for total respiratory, pneumonia and COPD in four largest cities in Korea using a temperature-matched case-crossover study design. ${ }^{12}$ Zhan et $a l^{28}$ used the National Morbidity, Mortality, and Air Pollution Study data from 106 communities of the USA during 1987-2000 to investigate the effect of temperature change between neighbouring days (TCNs) on mortality and found that prominent effects of TCNs on mortality for total respiratory, pneumonia and COPD diseases.

Temperature variability over a longer time duration (eg, intraseasonal), however, has rarely been studied for its potential health effects. We identified three epidemiological studies that had previously examined the health impacts of seasonal temperature variability, measured as the SD of intraseasonal temperature, and all of these studies focused on mortality. ${ }^{31-33}$ To our knowledge, this is the first study to link seasonal temperature variability with incident respiratory hospitalisations; thus, our findings may enrich our understanding towards the health impacts of seasonal temperature variability.

In our study, we found a positive association between wintertime temperature variability and incident respiratory diseases. This is consistent with previous studies using mortality as the health outcome. For example, Shi $e t a l^{32}$ reported that for each $1^{\circ} \mathrm{C}$ increase in SD of winter temperature associated with $4.1 \%$ (95\% CI 3.0\% to 5.2\%) increases in annual deaths using Medicare data with 2.7 million residents aged 65 years and older for the years 2000-2008 in the New England region of the USA.

Summertime temperature variability was not associated with incident respiratory diseases in our analyses, which is inconsistent with previous studies. ${ }^{31} 33$ This difference may be due to differences in city climates. Multicity studies have suggested that warm regions or areas with moderate winter climates have more significant cold effects than hot effects. ${ }^{34} 35$ This phenomenon can be explained by long-term adaptation, as people in warm areas are generally more sensitive to cold weather. ${ }^{36}$ Hong Kong has a subtropical climate, of which the summer is hot and humid. Epidemiological studies have suggested that heat effects in Hong Kong are not that evident, ${ }^{143738}$ possibly due to extensive use of air conditioning.

The biological mechanisms linking seasonal temperature variability with incident respiratory diseases have not been elucidated, although plausible explanations have been postulated. Temperature variability has been shown to affect the immune system's capability to resist infectious agents and cause inflammatory nasal responses in patients with persistent allergic rhinitis, which may trigger respiratory events. ${ }^{39}{ }^{40}$ Also, exposure to seasonal temperature variability has been reported to impede one's ability to adapt to local climate, which may increase the likelihood of adverse health outcomes like respiratory diseases. ${ }^{412}$ For example, locations with larger seasonal temperature variability produced stronger associations between daily temperature and mortality. ${ }^{35}{ }^{43}$

We found greater effects of wintertime temperature variability among females, which is consistent with previous shortterm temperature variability studies. ${ }^{44-46}$ The reason for that is probably due to biological difference. We found elders with lower personal monthly expenditure were more sensitive to increased wintertime temperature variability. Low personal monthly expenditure is regarded as an indicator of low socioeconomic position. The increased vulnerability of people with low socioeconomic status may be related to poor baseline health status, limited access to healthcare and poor living conditions. ${ }^{46} 47$

This study has some limitations. First, we obtained first occurrence of hospital admissions for respiratory diseases after enrolment. Respiratory disease hospitalisations prior to enrolment were not available so we could not identify participants who previously had respiratory diseases. However, sensitivity analysis excluding the participants with self-reported COPD/asthma at baseline gave similar estimates, which confirmed the robustness of our findings. Second, participants were volunteers; all residents aged 65 years or older were eligible to enrol. Thus, the participants in this study cohort could be more health conscious than the rest of the elderly population in Hong Kong. ${ }^{13}$ Third, the observed association between seasonal temperature variability and respiratory disease admissions might relate to the residents' perception of temperature modified by humidity and wind chill, for which not any fine resolution spatial data are available yet. Finally, individual household adaptation behaviours, such as heater or air conditioner usage, may affect the residents' personal exposure to seasonal temperature variability, measured only by outdoor weather stations. Although we did include house type in the regression model, it is still possible that the observed associations might be affected by temperature exposure misclassification. Despite these limitations, this is one of the few studies to examine the impacts of seasonal temperature variability on hospitalisations. Our findings might help better understand the impacts of climate change.

In conclusion, this study provides evidence that wintertime temperature variability increases the risks of incident total respiratory, pneumonia and COPD in older people. These findings should help better understand the health impacts of climate change.

Acknowledgements We would like to thank The Elderly Health Service of the Department of Health for providing the cohort data, the Hospital Authority for providing the hospital admission data and the Environmental Protection Department for providing the air pollution data.

Contributors FL and LT contributed to the conception and design and interpreted the results; RSL and CMW collected the cohort data; SS and HQ conducted statistical analyses; SS, FL, JEH, YW and LT drafted the manuscript for important intellectual content. All authors critically reviewed and accepted the final version of the manuscript.

Funding The authors have not declared a specific grant for this research from any funding agency in the public, commercial or not-for-profit sectors.

Competing interests None declared.

Patient consent Not required.

Ethics approval Ethics approval was obtained from the Ethics Committee of the Faculty of Medicine, The University of Hong Kong and of the Department of Health of Hong Kong.

Provenance and peer review Not commissioned; externally peer reviewed.

(c) Article author(s) (or their employer(s) unless otherwise stated in the text of the article) 2018. All rights reserved. No commercial use is permitted unless otherwise expressly granted.

\section{REFERENCES}

1 Ruuskanen O, Lahti E, Jennings LC, et al. Viral pneumonia. Lancet 2011;377:1264-75.

2 World Health Organization. The top 10 causes of death 2017. http://www.who.int/ mediacentre/factsheets/fs310/en/ (accessed 5 Jun 2017).

3 Haines A, Kovats RS, Campbell-Lendrum D, et al. Climate change and human health: impacts, vulnerability and public health. Public Health 2006;120:585-96. 
4 Meehl GA, Tebaldi C. More intense, more frequent, and longer lasting heat waves in the 21st century. Science 2004;305:994-7.

5 Rahmstorf S, Coumou D. Increase of extreme events in a warming world. Proc Natl Acad Sci U SA 2011;108:17905-9.

6 Stocker T. Climate change 2013: the physical science basis: Working Group I contribution to the Fifth assessment report of the Intergovernmental Panel on Climate Change. Cambridge: Cambridge University Press, 2014.

7 Coumou D, Rahmstorf S. A decade of weather extremes. Nat Clim Chang 2012;2:491-6.

8 Ayres JG, Forsberg B, Annesi-Maesano I, et al. Climate change and respiratory disease: European Respiratory Society position statement. Eur Respir J 2009;34:295-302.

9 Mirsaeidi M, Motahari H, Taghizadeh Khamesi M, et al. Climate change and respiratory infections. Ann Am Thorac Soc 2016;13:1223-30.

10 Group TE. Cold exposure and winter mortality from ischaemic heart disease, cerebrovascular disease, respiratory disease, and all causes in warm and cold regions of Europe. The Eurowinter Group. Lancet 1997;349:1341-6.

11 Lam HC, Li AM, Chan EY, et al. The short-term association between asthma hospitalisations, ambient temperature, other meteorological factors and air pollutants in Hong Kong: a time-series study. Thorax 2016;71:1097-109.

$12 \mathrm{Lim}$ YH, Hong YC, Kim H. Effects of diurnal temperature range on cardiovascular and respiratory hospital admissions in Korea. Sci Total Environ 2012;417418:55-60.

13 Schooling CM, Chan WM, Leung SL, et al. Cohort Profile: Hong Kong Department of Health Elderly Health Service Cohort. Int J Epidemio/ 2016;45:64-72.

14 Tian L, Qiu H, Sun S, et al. Emergency Cardiovascular Hospitalization Risk Attributable to Cold Temperatures in Hong Kong. Circ Cardiovasc Qual Outcomes 2016;9:135-42.

15 Jarvis CH, Stuart N. A Comparison among Strategies for Interpolating Maximum and Minimum Daily Air Temperatures. Part II: The Interaction between Number of Guiding Variables and the Type of Interpolation Method. Journal of Applied Meteorology 2001;40:1075-84.

16 Tabios GQ, Salas JD. A comparative analysis of techniques for spatial interpolation of precipitation. J Am Water Resour Assoc 1985;21:365-80.

17 Deutsch CV, Journel AG. Geostatistical software library and user's guide. New York: Oxford University Press, 1992;119:147.

18 NASA (National Aeronautics and Space Administration). NASA's Earth Observing System Homepage. Washington, DC: NASA (National Aeronautics and Space Administration), 2013.

19 Li C, Akh L, Mao J, et al. Retrieval, validation, and application of the 1-km aerosol optical depth from MODIS measurements over Hong Kong. IEEE Trans Geosci Remote Sens 2005;43:2650-8.

20 Wong CM, Lai HK, Tsang H, et al. Satellite-Based Estimates of Long-Term Exposure to Fine Particles and Association with Mortality in Elderly Hong Kong Residents. Environ Health Perspect 2015;123:1167-72.

21 Wong CM, Tsang H, Lai HK, et al. Cancer Mortality Risks from Long-term Exposure to Ambient Fine Particle. Cancer Epidemiol Biomarkers Prev 2016;25:839-45.

22 Qiu $\mathrm{H}$, Sun S, Tsang $\mathrm{H}$, et al. Fine particulate matter exposure and incidence of stroke: A cohort study in Hong Kong. Neurology 2017;88:1709-17.

23 Wong CM, Ou CQ, Chan KP, et al. The effects of air pollution on mortality in socially deprived urban areas in Hong Kong, China. Environ Health Perspect 2008:116:1189-94.

24 Wong CM, Hedley AJ, Lam TH, et al. Estimation of variations in health care needs between small areas in HK using routine statistics on morbidity, mortality and socioeconomic and demographic characteristics of the population. Hong Kong: Health, Welfare and Food Bureau, 1999
25 Andersen PK, Gill RD. Cox's Regression Model for Counting Processes: A Large Sample Study. The Annals of Statistics 1982;10:1100-20.

26 Buckley JP, Samet JM, Richardson DB. Commentary: Does air pollution confound studies of temperature? Epidemiology 2014;25:242-5.

27 Wang MZ, Zheng S, He SL, et al. The association between diurnal temperature range and emergency room admissions for cardiovascular, respiratory, digestive and genitourinary disease among the elderly: a time series study. Sci Total Environ 2013;456-457:370-5.

28 Zhan Z, Zhao Y, Pang S, et al. Temperature change between neighboring days and mortality in United States: A nationwide study. Sci Total Environ 2017;584585:1152-61.

29 Liang WM, Liu WP, Kuo HW. Diurnal temperature range and emergency room admissions for chronic obstructive pulmonary disease in Taiwan. Int J Biometeorol 2009;53:17-23.

30 Lim YH, Reid CE, Mann JK, et al. Diurnal temperature range and short-term mortality in large US communities. Int J Biometeorol 2015;59:1311-9.

31 Shi L, Kloog I, Zanobetti A, et al. Impacts of Temperature and its Variability on Mortality in New England. Nat Clim Chang 2015;5:988-91.

32 Shi L, Liu P, Wang Y, et al. Chronic effects of temperature on mortality in the Southeastern USA using satellite-based exposure metrics. Sci Rep 2016;6:30161.

33 Zanobetti A, O'Neill MS, Gronlund CJ, et al. Summer temperature variability and long-term survival among elderly people with chronic disease. Proc Natl Acad Sci U S A 2012;109:6608-13.

34 Medina-Ramón M, Schwartz J. Temperature, temperature extremes, and mortality: a study of acclimatisation and effect modification in 50 US cities. Occup Environ Med 2007;64:827-33

35 Braga AL, Zanobetti A, Schwartz J. The effect of weather on respiratory and cardiovascular deaths in 12 U.S. cities. Environ Health Perspect 2002:110:859-63.

36 Anderson BG, Bell ML. Weather-related mortality: how heat, cold, and heat waves affect mortality in the United States. Epidemiology 2009;20:205-13.

37 Yi W, Chan AP. Effects of temperature on mortality in Hong Kong: a time series analysis. Int J Biometeorol 2015;59:927-36.

38 Sun S, Tian L, Qiu H, et al. The influence of pre-existing health conditions on shortterm mortality risks of temperature: Evidence from a prospective Chinese elderly cohort in Hong Kong. Environ Res 2016:148:7-14.

39 Bull GM. The weather and deaths from pneumonia. Lancet 1980;1:1405-8.

40 Graudenz GS, Landgraf RG, Jancar S, et al. The role of allergic rhinitis in nasal responses to sudden temperature changes. J Allergy Clin Immunol 2006:118:1126-32.

41 Nielsen B, Hales JR, Strange S, et al. Human circulatory and thermoregulatory adaptations with heat acclimation and exercise in a hot, dry environment. J Physiol 1993:460:467-85.

42 Lee $M$, Nordio F, Zanobetti A, et al. Acclimatization across space and time in the effects of temperature on mortality: a time-series analysis. Environ Health 2014;13:89.

43 Braga AL, Zanobetti A, Schwartz J. The time course of weather-related deaths. Epidemiology 2001;12:662-7.

$44 \mathrm{Lim}$ YH, Park AK, Kim H. Modifiers of diurnal temperature range and mortality association in six Korean cities. Int J Biometeorol 2012:56:33-42.

45 Zhou X, Zhao A, Meng X, et al. Acute effects of diurnal temperature range on mortality in 8 Chinese cities. Sci Total Environ 2014:493:92-7.

46 Yang J, Liu HZ, Ou CQ, et al. Global climate change: impact of diurnal temperature range on mortality in Guangzhou, China. Environ Pollut 2013;175:131-6.

47 O'Neill MS, Zanobetti A, Schwartz J. Modifiers of the temperature and mortality association in seven US cities. Am J Epidemiol 2003;157:1074-82. 